\title{
Spin Reorientation in Substituted Barium Ferrite
}

\author{
J.M. Greneche, N. Randrianantoandro, Y. Labaye, F. Boust*, E. Flavin* and H. Pascard** \\ Laboratoire de Physique de l'État Condensé, URA 807 du CNRS, Université du Maine, 72017 Le Mans \\ cedex, France \\ * Laboratoire Matériaux OM, ONERA, 29 avenue de la Division Leclerc, 92322 Châtillon cedex, France \\ ** Laboratoire des Solides Irradiés, URA 1380 du CNRS, École Polytechnique, 91128 Palaiseau cedex, \\ France
}

\begin{abstract}
The structural transformation of substituted hexagonal ferrite powders $\mathrm{BaFe}_{10} \mathrm{CoTiO}_{19}$ prepared by mechanical milling, was investigated as a function of grinding time by means of Mössbauer spectrometry and static magnetic measurements. A spin reorientation is clearly evidenced from the temperature dependence of the hyperfine parameters and of the initial magnetic permeability on the powder mechanically treated for $23 \mathrm{~h}$.
\end{abstract}

\section{INTRODUCTION}

Hexagonal ferrites always exhibit great technological interest as permanent magnets and high frequency devices. So, we investigated the electromagnetic properties of $\mathrm{Co}^{2+}$ and $\mathrm{Ti}^{4+}$ substituted baryum ferrite powders with various grain sizes, resulting from mechanical milling. It is well established that the effect of milling is not only to decrease the grain size but also to modify the crystalline and the microcrystalline structures and consequently the magnetic properties (see [1] for the strontium hexagonal ferrite). Indeed, the mechanical treatment first divides the powder grains into ultrafine particles, and then induces structural distortions preferentially located at the vicinity of the surface and at the boundaries of nanometric domains, leading to a nanostructured powders [2].

In the present study, the bulk material with chemical composition $\mathrm{BaFe}_{10} \mathrm{CoTiO}_{19}$, was reduced to small particles by dry ball milling, for different milling times ( $1 \mathrm{~h}-2 \mathrm{~h}-3 \mathrm{~h}-6 \mathrm{~h}$ and $23 \mathrm{~h})$. The crystallographic and magnetic transformations induced by ball milling are investigated by static magnetic measurements and Mössbauer spectrometry performed as a function of temperature.

\section{RESULTS}

Mössbauer experiments were carried out in transmission geometry over the temperature range $4.2 \mathrm{~K}-650 \mathrm{~K}$, using a constant acceleration spectrometer and a ${ }^{57} \mathrm{Co}$ source diffused into a rhodium matrix. Three different regimes can be clearly distinguished from the temperature dependence of Mössbauer spectra recorded on $\mathrm{BaFe}_{10} \mathrm{CoTiO}_{19}$ powders mechanically treated for 23h: (i) below 150K, the spectra consist of magnetic sextets with rather well-resolved lines; (ii) for $150 \mathrm{~K}<\mathrm{T}<$ $620 \mathrm{~K}$, the spectra can be decomposed in two components: a central paramagnetic doublet with a proportion which increases with the temperature and a broadened magnetic sextet; (iii) above $T=620 \mathrm{~K}$, the spectra reveal only the presence of pure electric hyperfine interactions. For $T<150 \mathrm{~K}$, the best fit requires the superimposition of four magnetic components: this description is consistent with the different iron sites present in the crystallographic structure. The values of the hyperfine field are weakly reduced by comparison to those observed from the unmilled $\mathrm{BaFe}_{10} \mathrm{CoTiO}_{19}$ sample [3], this decrease may be attributed to the cationic distribution surrounding $\mathrm{Fe}$ nuclei, induced by the $\mathrm{Co}^{2+}$ and $\mathrm{Ti}^{4+}$ substitutions. Then, we concentrated on the analysis of spectra recorded in the intermediate temperature range $(150 \mathrm{~K}<\mathrm{T}<600 \mathrm{~K})$ because of their rather complex hyperfine structure. They were fitted with a continuous and discrete distribution of static hyperfine fields superimposed to a quadrupolar contribution. Finally, the temperature dependence of the average hyperfine field is plotted figure 1, including (a) or not (b) the paramagnetic contribution. Curve (b) clearly shows the presence of a cusp at $\mathrm{T} \approx 220 \mathrm{~K}$. The temperature dependence of the paramagnetic fraction is also plotted on figure 1 (c); let us note that we assume the same recoil free fraction $f(L a m b-M o ̈ s s b a u e r)$ factor for both magnetic and quadrupolar components. 


\begin{tabular}{|c|c|c|c|c|c|c|}
\hline $\begin{array}{c}\mathrm{MT} \\
(\mathrm{h})\end{array}$ & 0 & 1 & 2 & 3 & 6 & 23 \\
\hline $\begin{array}{c}\mathrm{Ms} \\
(\mathrm{uem} / \mathrm{g})\end{array}$ & 68 & 63 & 60 & 57 & 52 & 36 \\
\hline $\begin{array}{c}\mathrm{Hc} \\
(\mathrm{Oe})\end{array}$ & 38 & 55 & 68 & 84 & 98 & 132 \\
\hline
\end{tabular}

Table 1: Saturation magnetization $\mathrm{Ms}$ and coercive field $\mathrm{Hc}$ for applied magnetic fields of $20 \mathrm{~T}$ and $0.35 \mathrm{~T}$, respectively measured at $300 \mathrm{~K}$, on $\mathrm{BaFe}_{10} \mathrm{CoTiO}_{19}$ powders, for different milling times $M T$.
Both saturation magnetization and coercive field were measured at $300 \mathrm{~K}$ on $\mathrm{BaFe}_{10} \mathrm{CoTiO}_{19}$ powders mechanically treated for different times (see table 1): a strong decrease of the saturation magnetization and a large increase of the coercive field are observed with increasing grinding times. The temperature dependence of the initial magnetic permeability was measured on a toroidal powdered sample of $\mathrm{BaFe}_{10} \mathrm{COTiO}_{19}$ mechanically treated for $23 \mathrm{~h}$ and compared to that of the unmilled sample, as it is shown on figure 2. The low temperature maximum ( $\operatorname{Tr} \approx$ $250 \mathrm{~K}$ ) corresponds to a spin reorientation due to a change of magnetocrystalline anisotropy: below $\mathrm{Tr}$, the uniaxial anisotropy is oriented along $c$ axis, while above $\mathrm{Tr}$, the planar anisotropy is perpendicular to $c$ axis.

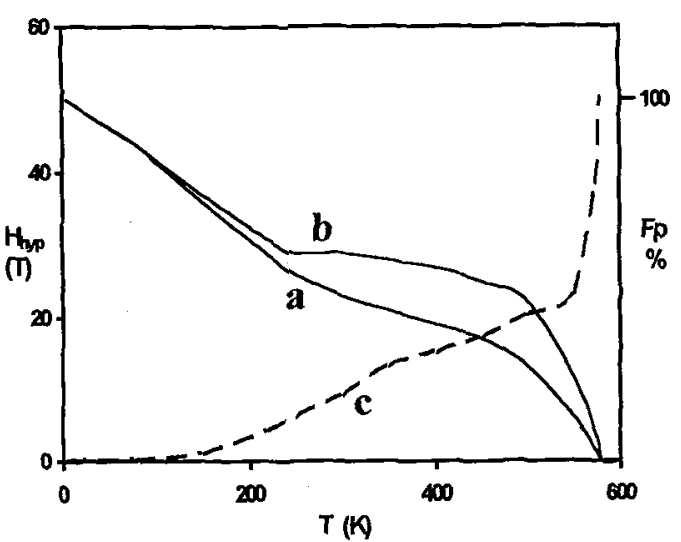

Figure 1: Temperature dependencies of the average hyperfine field for the $\mathrm{BaFe}_{10} \mathrm{CoTiO}_{19}$ powder after a mechanical milling of $23 \mathrm{~h}$, including (a) or not (b) the paramagnetic contribution and of the paramagnetic fraction (c).

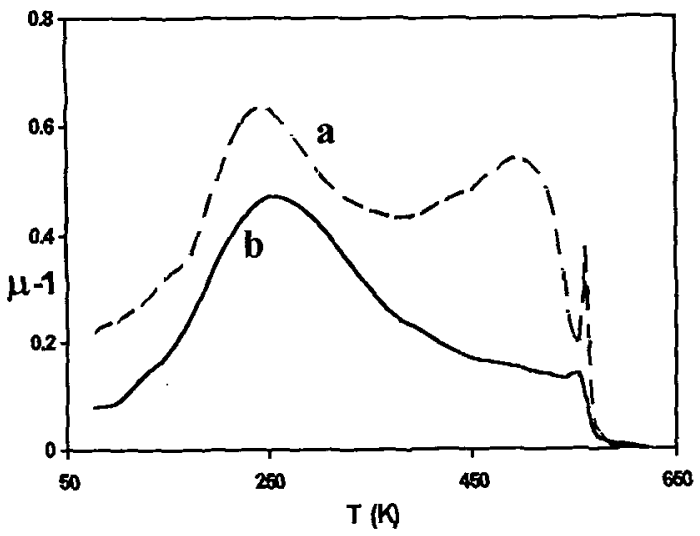

Figure 2: Temperature dependence of the initial magnetic permeability of the $\mathrm{BaFe}_{10} \mathrm{CoTiO}_{19}$ powder before mechanical milling (a) and after a mechanical milling of $23 \mathrm{~h} \mathrm{(b)}$.

\section{DISCUSSION}

The hyperfine structure and magnetic characteristics described above are related to the nanostructured behaviour of the powder induced by the dry ball milling process. At low temperatures, the lowering of the average hyperfine field between unmilled and mechanically treated powders, is essentially due to the occurrence of disordered grain boundaries. In the intermediate temperature range, the cusp observed at $\approx 220 \mathrm{~K}$ can be interpreted in terms of a spin reorientation, in agreement with initial magnetic permeability data. At higher temperatures, the progressive occurrence of a paramagnetic contribution is probably significant of both the existence of highly disordered or amorphous zones and the presence of superparamagnetic effects induced either by a distribution of nanocrystallites of different sizes, or by a superficial contribution. In order to check the existence of an amorphous phase, the powder mechanically treated for $23 \mathrm{~h}$ was annealed during $24 \mathrm{~h}$ at $1230^{\circ} \mathrm{C}$. The $300 \mathrm{~K}-$ Mössbauer spectrum is similar to that obtained on the unmilled powder, which is consistent with previous studies performed on mechanically milled strontium hexaferrite [1].

\section{References}

[1] U. Heinecke, C. Cruz, E. Wieser, C. Bernhardt, Phys. Stat. Sol. (a) 77 (1983) 225

[2] H. Gleiter, Prog. Mat. Sci. 33 (1989) 223

[3] X. Z. Zhou, A.H. Morrish, Z. W. Li, IEEE Trans Magn 27 (1992) 6 